\title{
A Weyl Group for the Virasoro and $N=1$ Super-Virasoro Algebras
}

\author{
V. K. Dobrev * \\ International Centre for Theoretical Physics, Trieste, Italy
}

\begin{abstract}
We introduce a Weyl group for the highest weight modules over the Virasoro algebra and the Neveu-Schwarz and Ramond superalgebras. Using this group we rewrite the character formulae for the irreducible highest weight modules over these algebras in the form of the classical Weyl character formula for the finite-dimensional irreducible representations of semi-simple Lie algebras (and also of the Weyl-Kac character formula for the integrable highest weight modules over affine Kac-Moody algebras). This is the same group we introduced recently in order to rewrite in a similar manner the characters of the singular highest weight modules over the affine Kac-Moody algebra $A_{1}^{(1)}$.
\end{abstract}

\section{Introduction}

The present paper is an attempt to extend the notion of Weyl group to the Virasoro and $N=1$ super-Virasoro algebras. As we know Weyl groups and their generalizations are very important in the representation theory of semi-simple Lie algebras $\mathfrak{G}_{0}$, of affine Kac-Moody algebras $\mathfrak{G}^{\mathfrak{5}}$ and of super-algebras $\mathfrak{G}_{S}$ associated with (generalized) Cartan matrices (see e.g. [1-5]). In the generic cases the Weyl(-Kac) group is essential in the formulae describing the characters of the irreducible finitedimensional representations of $\mathfrak{b}_{0}$, of the integrable representations of $\mathfrak{G}$ and of the typical representations of $\tilde{\mathfrak{G}}_{S}[1-5]$. Nevertheless, there are large and interesting classes of representations for which the Weyl(-Kac) group does not play a similar role. Examples of these are the singular representations of affine KacMoody algebras $[6,7]$ characterized by the fact that the central charge is equal to the dual coxeter number and the atypical representations of finite-dimensional $\mathfrak{b}_{S}$. Recently [8], using results of [9] we derived character formulae for singular highest weight modules over $A_{1}^{(1)}$. Then we introduced new Weyl groups $W_{a}, W_{a}^{+}$, which we used to rewrite these character formulae so that they look exactly as the usual Weyl(-Kac) formulae with the Weyl(-Kac) group $W$ replaced by $W_{a}$ or $W_{a}^{+}$.

* Permanent address: Institute of Nuclear Research and Nuclear Energy, Bulgarian Academy of Sciences, 72, Boul. Lenin, BG-1784 Sofia, Bulgaria 
A priori it is not clear whether the notion of a Weyl group can be generalized to the (super-)Virasoro algebras since these algebras do not have (generalized) Cartan matrices. We do this in the present paper. It turns out that the Weyl group $W_{a}$ of [8] can play the same role in describing the characters of the irreducible highest weight modules $L$ over the Virasoro and $N=1$ super-Virasoro algebras. The only difference is that one needs also the notion of $V$-active elements of $W_{a}$, where $V$ are the Verma modules with quotients $L$.

The paper is organized as follows. Section 1 and the two tables summarize the necessary information on the Virasoro, and the $N=1$ super-Virasoro algebras (that is the Neveu-Schwarz and Ramond superalgebras) and their representations. Section 2 recalls the character formulae for $L$. Section 3 contains our main result.

\section{Preliminaries}

The Virasoro algebra [10] $\hat{W}$ is a complex Lie algebra with basis $z, L_{i}, i \in \mathbb{Z}$ and Lie brackets:

$$
\left[L_{i}, L_{j}\right]=(i-j) L_{i+j}+\frac{z}{12}\left(i^{3}-i\right) \delta_{i,-j}, \quad\left[z, L_{i}\right]=0
$$

The $N=1$ super-Virasoro algebras are the Neveu-Schwarz and Ramond superalgebras.

The Neveu-Schwarz superalgebra [11] $\hat{S}$ is a complex Lie superalgebra with basis $z, L_{i}, i \in \mathbb{Z}, I_{\alpha}, \alpha \in \mathbb{Z}+\frac{1}{2}$ and Lie brackets

$$
\begin{gathered}
{\left[L_{i}, L_{j}\right]=(i-j) L_{i+j}+\frac{z}{8}\left(i^{3}-i\right) \delta_{i,-j},} \\
{\left[I_{\alpha}, I_{\beta}\right]=2 L_{\alpha+\beta}+\frac{z}{2}\left(\alpha^{2}-\frac{1}{4}\right) \delta_{\alpha,-\beta},} \\
{\left[L_{i}, I_{\alpha}\right]=\left(\frac{i}{2}-\alpha\right) I_{i+\alpha}, \quad\left[z, L_{i}\right]=0, \quad\left[z, I_{\alpha}\right]=0 .}
\end{gathered}
$$

The Ramond superalgebra [12] $\hat{R}$ is a complex Lie superalgebra with basis $z, L_{i}$, $i \in \mathbb{Z}, I_{\alpha}, \alpha \in \mathbb{Z}$ and Lie brackets given again by (2).

Further $\hat{Q}$ will denote $\hat{W}, \hat{S}$ or $\hat{R}$ when a statement holds for all three algebras. The elements $z, L_{i}$ are even, and $I_{\alpha}$ are odd. The grading of $\hat{Q}$ is given by $\operatorname{deg} z=0$, $\operatorname{deg} L_{i}=i, \operatorname{deg} I_{\alpha}=\alpha$. We have the decomposition

$$
\widehat{Q}=\hat{Q}_{+} \oplus \hat{Q}_{0} \oplus \hat{Q}_{-},
$$

where $W_{ \pm}$are generated by $L_{ \pm i}, i=1,2, \hat{S}_{ \pm}$are generated by $I_{ \pm \alpha}, \alpha=\frac{1}{2}, \frac{3}{2} ; \hat{R}_{ \pm}$are generated by $L_{ \pm 1}, I_{ \pm 1}$; and $\hat{W}_{0}$ and $\hat{S}_{0}$ are spanned by $L_{0}$ and $z ; \hat{R}_{0}$ is spanned by $z$, $L_{0}$, and $I_{0}$.

Besides highest weight modules (HWM) we shall use generalized highest weight modules in the $\hat{R}$ case because of the relation $L_{0}=\left(I_{0}\right)^{2}+z / 16$. 
A generalized highest weight module over $\hat{Q}$ is characterized by its highest weight $\lambda \in Q_{0}^{*}$ and generalized highest weight vector $\tilde{v}_{0}$ such that

$$
\begin{gathered}
L_{i} \tilde{v}_{0}=0, \quad i>0, \quad \text { for } \hat{Q}, \\
I_{\alpha} \tilde{v}_{0}=0, \quad \alpha>0, \quad \text { for } \hat{S}, \hat{R}, \\
L_{0} \tilde{v}_{0}=\lambda\left(L_{0}\right) \tilde{v}_{0}=h \tilde{v}_{0}, \quad h \in \mathbb{C}, \quad \text { for } \hat{Q}, \\
z \tilde{v}_{0}=\lambda(z) \tilde{v}_{0}=c \tilde{v}_{0}, \quad c \in \mathbb{C}, \quad \text { for } \hat{Q} .
\end{gathered}
$$

A generalized highest weight vector $\tilde{v}_{0}$ can be defined in the $\hat{R}$ case as the direct sum $I_{0} v_{0} \oplus v_{0}$, where $v_{0}$ fulfills all conditions (4), (5), i.e., it is a usual highest weight vector. For $\hat{W}, \hat{S}, \tilde{v}_{0}=v_{0}$ so generalized HWM (GHWM) for $\hat{W}, \hat{S}$ are usual HWM.

Further we shall work with the so-called (generalized) Verma modules over $\hat{Q}$. A (generalized) Verma module $V^{\lambda}=V^{h, c}$ is an induced GHWM with highest weight $\lambda$ such that $V^{\lambda} \cong \mathscr{U}\left(\hat{Q}_{-}\right) \otimes \tilde{v}_{0}$, where $\mathscr{U}\left(\hat{Q}_{-}\right)$is the universal enveloping algebra of $\hat{Q}_{-}$. ( $V^{\lambda}$ is the largest GHWM with highest weight $\lambda$ as every other GHWM with the same highest weight may be obtained as a factor module of $V^{\lambda}$.) It is known (see $[13-15]$ for $\hat{W},[14,16]$ for $\widehat{S},[17,16]$ for $\hat{R})$ that $V^{\lambda}=V^{h, c}=V^{h, c}$ is reducible iff $h$ and $c$ are related as follows:

$$
\text { either } h=h_{(m, n)}=h_{0}+\frac{1}{4}\left(m \alpha_{+}+n \alpha_{-}\right)^{2}+\frac{1}{8} \mu, \quad \text { for } \hat{Q},
$$

where $m, n \in \frac{1}{v} \mathbb{N}$,

$$
\begin{gathered}
v= \begin{cases}1 & \text { for } \hat{W}, \\
\frac{1}{2} & \text { for } \hat{S}, \hat{R},\end{cases} \\
m-n \in \mathbb{Z}+\mu, \quad \text { for } \hat{Q}, \\
\mu= \begin{cases}0 & \text { for } \hat{W}, \hat{S}, \\
\frac{1}{2} & \text { for } \hat{R},\end{cases} \\
h_{0}= \begin{cases}\frac{c-1}{24} & \text { for } \hat{W}, \\
\frac{c-1}{16} & \text { for } \hat{S}, \hat{R}, \\
\alpha_{ \pm}=\left\{\begin{array}{l}
\frac{1}{\sqrt{24}}(\sqrt{1-c} \pm \sqrt{25-c}), \\
\frac{1}{2}(\sqrt{1-c} \pm \sqrt{9-c}),
\end{array} \text { for } \hat{W}, \hat{S},\right.\end{cases} \\
\text { or } h=\frac{c}{16} \text { for } \hat{R} .
\end{gathered}
$$

It is known [13]-[17] that the reducible $V=V^{h, c}, h=h_{(m, n)}$ contains a proper submodule isomorphic to the (generalized) Verma module $V^{\prime}=V^{h+v n m, c}$. In other words there exists a non-trivial embedding map between $V^{\prime}$ and $V$. These embedding maps are realized by the so-called singular vectors. A singular vector 
$v_{s} \in V$ is such that $v_{s} \neq \tilde{v}_{0}$ and $v_{s}$ has the property of the highest weight vector $\tilde{v}_{0}^{\prime}$ of $V^{\prime}$. More than this $v_{s}$ can be expressed, as an element of $\mathscr{U}\left(\hat{Q}_{-}\right) \tilde{v}_{0}$ by

$$
v_{s}=\mathscr{P}\left(\hat{Q}_{-}\right) \tilde{v}_{0}^{\prime}, \quad V^{\prime} \cong \mathscr{U}\left(\hat{Q}_{-}\right) \tilde{v}_{0}^{\prime} \cong \mathscr{U}\left(\hat{Q}_{-}\right) \mathscr{P}\left(\hat{Q}_{-}\right) \hat{v}_{0},
$$

where $\mathscr{P}\left(\hat{Q}_{-}\right)$is a homogeneous polynomial in $\mathscr{U}\left(\hat{Q}_{-}\right)$of degree $v m n$. (Here we should note that for superalgebras the maps under consideration are not embedding maps in general. This may happen if $\mathscr{P}\left(\hat{Q}_{-}\right)^{2}=0$, then $\mathscr{U}\left(\hat{Q}_{-}\right) \mathscr{P}\left(\hat{Q}_{-}\right) \tilde{v}_{0} \neq \mathscr{U}\left(\hat{Q}_{-}\right) \tilde{v}_{0}^{\prime}$. It cannot happen if there does not exist an element $X \in \mathscr{U}\left(\hat{Q}_{-}\right)$such that $X^{2}=0$. This is the case for $\hat{Q}$ and thus all maps in consideration are embeddings.)

In the case $h=c / 16$ for $\hat{R}$ the reducible $V=V^{c / 16, c}$ contains a proper submodule which is isomorphic to an ordinary Verma module $\widetilde{V}=\widetilde{V}^{c / 16, c}$ with the same highest weight and highest weight vector $\tilde{v}_{0}^{\mathrm{s}}=I_{0} v_{0} \oplus 0=I_{0} v_{0}$. In order to see this consider first $P \tilde{v}_{0}=P I_{0} v_{0} \oplus P v_{0}$, where $P \in \mathscr{U}\left(\hat{R}_{-}\right)$is a homogeneous element and the action of $X \in \hat{R}$ on $P \tilde{v}_{0}$,

$$
\begin{aligned}
X P \tilde{v}_{0}=\left(P^{\prime}+Q^{\prime} I_{0}\right) \tilde{v}_{0} & =\left(P^{\prime}+Q^{\prime} I_{0}\right) I_{0} v_{0} \oplus\left(P^{\prime}+Q^{\prime} I_{0}\right) v_{0} \\
& =\left(P^{\prime}+Q^{\prime}\right) I_{0} v_{0} \oplus\left(P^{\prime}+Q^{\prime}\left(L_{0}-z / 16\right)\right) v_{0} \\
& =\left(P^{\prime}+Q^{\prime}\right) I_{0} v_{0} \oplus\left(P^{\prime}+(h-c / 16) Q^{\prime}\right) v_{0},
\end{aligned}
$$

where $P^{\prime}, Q^{\prime} \in \mathscr{U}\left(\hat{R}_{-}\right)$; actually $P^{\prime}, Q^{\prime}$ are zero if $\operatorname{deg} X>|\operatorname{deg} P|$ and the action of $L_{0}, z$ on $\tilde{v}_{0}$ is accounted for in $P^{\prime}, Q^{\prime}$. Analogously $P \tilde{v}_{0}^{s}=P I_{0} v_{0}$ and let $h=c / 16$ :

$$
\begin{aligned}
X P \tilde{v}_{0}^{s} & =\left(P^{\prime}+Q^{\prime} I_{0}\right) \tilde{v}_{0}^{s}=\left(P^{\prime}+Q^{\prime} I_{0}\right) I_{0} v_{0} \\
& =P^{\prime} I_{0} v_{0}+Q^{\prime}\left(L_{0}-z / 16\right) v_{0}=P^{\prime} I_{0} v_{0}=P^{\prime} \tilde{v}_{0}^{s} \in \mathscr{U}\left(\hat{R}_{-}\right) \tilde{v}_{0}^{s} .
\end{aligned}
$$

Note that the factor module $V / \widehat{V}$ is isomorphic to $\tilde{V}$. Further in the case $h=c / 16$ we shall consider $\widetilde{V}$ instead of $V^{c / 16, c}$ for $\hat{R}$. It is also possible that (6) and (7) hold simultaneously; then

$$
h=\frac{c}{16}=h_{(n, m)}=\frac{c-1}{16}+\frac{1}{4}\left(m \alpha_{+}+n a_{-}\right)^{2}+\frac{1}{16},
$$

or,

$$
m \alpha_{+}+n \alpha_{-}=0
$$

or,

$$
-\alpha_{-} / \alpha_{+}=\frac{m}{n}=\frac{2 m}{2 n}=\frac{p}{q}, \quad p, q \in \mathbb{N}, \quad\left(m-n \in \mathbb{Z}+\frac{1}{2}\right) .
$$

In this case $\tilde{V}$ is further reducible and everything we said after formula (7) applies also to this case.

The reducible (together with some irreducible in some cases) GHWM are grouped into multiplets $([18,6,19,20,21,7])$. A set $\mathscr{M}$ of GHWM over a complex (infinite-dimensional) (super) Lie algebra is said to form a multiplet if: 1) $V \in \mathscr{M} \Rightarrow \mathscr{M} \supset \mathscr{M}_{V} \neq \emptyset$, where $\mathscr{M}_{V}$ is the set of all GHWM $V^{\prime} \neq V$ such that there exists a non-trivial embedding map between $V^{\prime}$ and $\left.V ; 2\right) \mathscr{M}$ does not contain proper subsets fulfilling 1). 
As usual we shall represent a multiplet $\mathscr{M}$ by a connected oriented graph. The vertices of the graph correspond to the GHWM of $\mathscr{M}$ and the arrows connecting the vertices correspond to those embedding maps which are not compositions of other embedding maps. The multiplets represented by the same graph are said to belong to one and the same type of multiplets $[6,7,18-21]$. Then we use parametrization to distinguish the multiplets belonging to a fixed type. Also in some cases it is useful to consider subtypes when there is no convenient parametrization for the whole type.

Let us summarize the results on the multiplet classification of the reducible (generalized) Verma modules for $\hat{W}$ [22] in the form given in [19] (cf. also [23]) and for $\hat{S}, \hat{R}[21]$. There are five types in each case denoted by $N^{0}, N_{+}^{1}, N_{-}^{1}, N_{+}^{2}, N_{-}^{2}$ which are shown in Table 1 . Note that in our convention $V \rightarrow V^{\prime}$ means that $V^{\prime}$ can be invariantly embedded in $V$ (arrows point to the embedded modules). We have also indicated the notation of [22] for $\hat{W}$.

The type $N^{0}$ occurs when $\alpha_{-} / \alpha_{+} \notin \mathbb{Q} \subset \mathbb{R}$. All other types occur when $\alpha_{-} / \alpha_{+} \in \mathbb{Q}$; then either $c \leqq 1$ or $c \geqq c_{+}$, where

$$
c_{+}=41-16 v= \begin{cases}25 & \text { for } \hat{W}, \\ 9 & \text { for } \hat{S}, \hat{R} .\end{cases}
$$

For $c<1$ we have type $N_{-}^{1}$ and subtypes $N_{-}^{21}, N_{-}^{22}$ of type $N_{-}^{2}$. In this case $-\alpha_{-} / \alpha_{+}$ $=p / q, p, q \in \mathbb{N}$ and then

$$
c=c_{p, q}^{-}=21-8 v-(10-4 v)\left(\frac{p}{q}+\frac{q}{p}\right)= \begin{cases}13-6\left(\frac{p}{q}+\frac{q}{p}\right) & \text { for } \hat{W}, \\ 5-2\left(\frac{p}{q}+\frac{q}{p}\right) & \text { for } \hat{S}, \hat{R} .\end{cases}
$$

For $c=1$ we have subtype $N_{-}^{23}$ of type $N_{-}^{2}$ (note $\left.c_{1,1}^{-}=1\right)$. For $c>c_{+}$we have type $N_{+}^{1}$ and subtypes $N_{+}^{21}, N_{+}^{22} ; \alpha_{-} / \alpha_{+}=\frac{p}{q}, p, q \in \mathbb{N}$ and

$$
c=c_{p, q}^{+}=21-8 v+(10-4 v)\left(\frac{p}{q}+\frac{q}{p}\right)= \begin{cases}13+6\left(\frac{p}{q}+\frac{q}{p}\right) & \text { for } \hat{W}, \\ 5+2\left(\frac{p}{q}+\frac{q}{p}\right) & \text { for } \hat{S}, \hat{R} .\end{cases}
$$

For $c=c_{+}$we have subtype $N_{+}^{23}$ (note that $c_{1,1}^{+}=c_{+}$).

The explicit parametrization of all types is given in Table 2. Note that in each case the types with $c \leqq 1$ have the same parametrization as those with $26-c \geqq c_{+}$. (For parametrization of subtypes see $[19,21]$.) We shall not need the explicit parametrization of the multiplets, i.e. the values of $h$ for the (generalized) Verma modules in Table 1 . We shall give only the values of $h$ for $V_{00}, V_{00}^{+}, V_{0}, V_{0}^{+}$:

$$
h=h_{(m, n)}=\frac{1}{4 v p q}\left[v^{2}(p n-q m)^{2}-(p-q)^{2}\right]+\frac{1}{8} \mu=h_{(\tilde{p}-m, \tilde{q}-n)}
$$

for $N_{-}^{1}, p, q, m, n$ are the parameters as of Table $2 ; h_{(m, n)}$ is the same quantity as in (6); note that for $\hat{R}$,

$$
h=h\left(\frac{p}{2}, \frac{q}{2}\right)=\frac{1}{16} c_{p, q}^{-}
$$


Table 1. Types of multiplets (embedding diagrams) of reducible Verma modules over the Virasoro $[22,19,23]$ and $N=1$ super-Virasoro [21] algebras (arrows point to the embedded modules)

$N^{0}$ (or II [22]) $V^{h, c} \longrightarrow V^{h+v m n, c}$
$N_{-}^{1}$ (or $\left.\mathrm{III}_{-}[22]\right) \quad V_{00}=V_{10}$
$N_{+}^{2}$ (or $\left.\mathrm{III}_{-}^{0}, \mathrm{III}_{+}^{00}[22]\right)$

Table 2. Parametrization of the types of multiplets of (generalized) Verma modules over the Virasoro [22,19] and $N=1$ super-Virasoro algebras [21]

\begin{tabular}{|c|c|c|c|}
\hline Type & $\begin{array}{l}\text { Param- } \\
\text { eters }\end{array}$ & $\begin{array}{l}\text { Range and constraints } \\
\text { on parameters }\end{array}$ & Remarks \\
\hline$N^{0}(\mathrm{II})$ & $c, m, n$ & $\begin{array}{c}c \in \mathbb{C}, m, n \in \frac{1}{v} \mathbb{N} \\
m-n \in \mathbb{Z}+\mu \\
\alpha_{-} / \alpha_{+} \notin \mathbb{Q},\end{array}$ & \\
\hline$N_{ \pm}^{1}\left(\mathrm{III}_{ \pm}\right)$ & $p, q, m, n$ & $\begin{array}{l}p, q \in \mathbb{N}, p<q, p X q \\
m, n \in \frac{1}{v} \mathbb{N}, \\
m-n \in \mathbb{Z}+\mu \\
p m \geqq q n, m<\tilde{q}\end{array}$ & $\begin{array}{l}\alpha_{-} / \alpha_{+}= \pm p / q ; \\
c=c_{p, q}^{ \pm} ; \\
\text {For } \hat{R} \text { and } N_{-}^{1} \\
(m, n)=(p / 2, q / 2) \text { is } \\
\text { the only possibility for } \tilde{V}^{c / 16, c}\end{array}$ \\
\hline$N_{ \pm}^{2}\left(\mathrm{III}_{ \pm}^{0}, \mathrm{III}_{ \pm}^{00}\right)$ & $p, q, n$ & $\begin{array}{l}0<n<\tilde{p} \neq \tilde{q}, n-\tilde{p} \in \mathbb{Z}+\mu \\
0=n<p \leqq q, \tilde{p} \in \mathbb{Z}+\mu \\
n=\tilde{p} \leqq \tilde{q}, \text { for } \hat{W}, \hat{S}\end{array}$ & $\begin{array}{l}\alpha_{-} / \alpha_{+}= \pm p / q ; \\
c=c_{p q}^{ \pm}\end{array} \begin{array}{l}p=q \Rightarrow p=q=1 \\
\Rightarrow \begin{cases}c=1 & \text { for } N_{-}^{2} \\
c=c_{+} & \text {for } N_{+}^{2}\end{cases} \\
\Rightarrow n=0, p=1 \text { for } \hat{W} \\
n=0, \tilde{p}=1 / 2 \text { for } \hat{R} \\
n=p=1 \text { for } \hat{W} \\
n=\tilde{p}=1 / 2 \text { for } \hat{S}\end{array}$ \\
\hline
\end{tabular}


is the only possibility for the reducible $\tilde{V}^{c / 16, c}$;

$$
h=h_{(m, n)}^{+}=\frac{1}{4 v p q}\left[(p+q)^{2}-v^{2}(p n-q m)\right]+\frac{1}{8} \mu\left(=\frac{1}{v}+\frac{1}{4} \mu-h_{(m, n)}\right)
$$

for $N_{+}^{1}$

$$
\begin{gathered}
h=h_{(m, \tilde{q})} \quad \text { for } N_{-}^{21}, \quad h=h_{(m, \tilde{q})}^{+} \text {for } N_{+}^{21}, \\
h=h_{(p, q)}\left(=h_{(\tilde{p}, \tilde{q})}\right) \text { for } N_{-}^{22}, \quad h=h_{(p, q)}^{+} \text {for } N_{+}^{22}, \\
h=h^{\varepsilon}=\frac{\varepsilon^{2}}{4 v}+\frac{\mu}{8} \text { for } N_{-}^{23}, \\
h^{\varepsilon+}=\frac{1}{v}+\frac{1}{4} \mu-h^{\varepsilon}=\frac{1}{v}+\frac{1}{8} \mu-\frac{\varepsilon^{2}}{4 v} \text { for } N_{+}^{23} .
\end{gathered}
$$

The interested reader can find the complete parametrization of all multiplets in [19] for $\hat{W}$ and in [21] for $\hat{S}, \hat{R}$.

\section{Characters of (Generalized) Verma Modules}

We recall the weight space decomposition of $V^{h, c}$

$$
V_{j}^{h, c}=\bigoplus_{j} V_{j}^{h, c}, \quad j \in \mathbb{Z}_{+} \quad \text { for } \hat{W}, \hat{R}, \quad j \in \frac{1}{2} \mathbb{Z}_{+} \quad \text { for } \hat{S},
$$

where $V_{j}^{h, c}$ are eigenspaces of $L_{0}$

$$
V_{j}^{h, c}=\left\{v \in V^{h, c} \mid L_{0} v=(h+j) v\right\} \cong \mathscr{U}\left(\hat{Q}_{-}\right)_{j} \tilde{v}_{0},
$$

where the last equality follows from

$$
\mathscr{U}\left(\hat{Q}_{-}\right)=\bigoplus_{j} \mathscr{U}\left(\hat{Q}_{-}\right)_{j}
$$

with the range of $j$ as in (16). For $\hat{R}$ we have also

$$
\begin{gathered}
V_{j}^{h, c}=V_{j, 1}^{h, c} \oplus V_{j, 2}^{h, c}, \\
V_{j, 1}^{h, c}=\mathscr{U}\left(\hat{Q}_{-}\right)_{j} I_{0} v_{0}, \quad V_{j, 2}^{h, c}=\mathscr{U}\left(\hat{Q}_{-}\right)_{j} v_{0} .
\end{gathered}
$$

The character of $V^{h, c}$ is defined (cf. $[13,14,17]$ ) as

$$
\operatorname{ch} V^{h, c}(t)=\sum_{j}\left(\operatorname{dim} V_{j}^{h, c}\right) t^{h+j}=t^{h} \sum_{j} p(j) t^{j}=t^{h} \psi(t),
$$

where $p(j)$ is the partition function $[\mathrm{p}(\mathrm{j})=$ \# of ways $j$ can be represented as the sum of positive integers (and half-integers for $\widehat{S}$ ); $p(0) \equiv 1]$ while $\psi(t)$ is given by $[13,14$, 17]:

$$
\psi(t)= \begin{cases}\prod_{k \in \mathbb{N}}\left(1-t^{k}\right)^{-1} & \text { for } \hat{W}, \\ \prod_{k \in \mathbb{N}}\left(1+t^{k-1 / 2}\right) /\left(1-t^{k}\right) & \text { for } \hat{S} \\ \prod_{k \in \mathbb{N}} 2\left(1+t^{k}\right) /\left(1-t^{k}\right) & \text { for } \hat{R} .\end{cases}
$$

The factor of 2 for $\hat{R}$ appears because of the relation

$$
\operatorname{dim} V_{j}^{h, c}=(1+2 \mu) \operatorname{dim} \mathscr{U}\left(\hat{Q}_{-}\right)_{j} .
$$


For $\hat{R}$ and $h=c / 16$ we have for $\hat{V}=\widetilde{V}^{c / 16, c} \equiv \mathscr{U}\left(\tilde{R}_{-}\right) I_{0} v_{0}$,

$$
\operatorname{ch} \tilde{V}(t)=t^{c / 16} \prod_{k \in \mathbb{N}}\left(1+t^{k}\right) /\left(1-t^{k}\right) .
$$

Further $L^{h, c}$ shall denote the unique irreducible quotient of $V^{h, c}$, such that $L^{h, c}$ $=V^{h, c} / I^{h, c}$, where $I^{h, c}$ is the maximal proper submodule of $V^{h, c}$. For $\hat{R}$ and $h=c / 16$, $\widetilde{V}=\tilde{V}^{c / 16, c}, \tilde{L}=\tilde{L}^{c / 16, c}=\widetilde{V} / \widetilde{I}, \tilde{I}$ is the maximal proper submodule of $\tilde{V}$, $\left(\tilde{V} \cong V^{c / 16, c} / \tilde{V}\right)$. If $V^{h, c}$ (respectively $\left.\tilde{V}\right)$ is irreducible, $L^{h, c}=V^{h, c}($ respectively $L=\tilde{V})$. If $V^{h, c}(\widetilde{V})$ is not irreducible then the character formula for $L^{h, c}(\widetilde{L})$ is more complicated than (22) [respectively (25)].

We recall the character formulae for $\widetilde{W}[24,23,25]$ (for partial results see $[13$, $14,26-30]$ ) and for $\hat{S}, \hat{R}[25]$ (for partial results see $[14,29,31]$ ) in a form suitable for our purposes.

In the $N^{0}$ case the embedded $V^{h+v m n, c}$ is irreducible while for $L^{h, c}$ using the results of [23] one can obtain

$$
\begin{aligned}
\operatorname{ch} L^{h, c} & =\operatorname{ch} V^{h, c}-\operatorname{ch} V^{h+v m n, c} \\
& =\left(1-t^{v m n}\right) \operatorname{ch} V^{h, c} .
\end{aligned}
$$

In the $N^{1}$ - case let us denote by $L_{0 k}, L_{1 k}, L_{0 k}^{\prime}, L_{1 k}^{\prime}$ the irreducible factor-modules of $V_{0 k}, V_{1 k}, V_{0 k}^{\prime}, V_{1 k}^{\prime}$ respectively, where $[19,21,25]$

$$
\begin{gathered}
V_{0 k}=V^{h+v k(\tilde{p} \tilde{q} k+\tilde{q} m-\tilde{p} n), c}, \quad V_{1 k}=V^{h+\nu k(\tilde{p} \tilde{q} k-\tilde{q} m+\tilde{p} n)}, \\
V_{0 k}^{\prime}=V^{h+\nu(\tilde{q} k+m)(\tilde{p} k+n), c}, \quad V_{1 k}^{\prime}=V^{h+v(\tilde{q} k+\tilde{q}-m)(\tilde{p} k+\tilde{p}-n), c},
\end{gathered}
$$

and $h=h_{(m, n)}$ is given by (13). Then we have [23], [24], [25], (22):

$$
\begin{gathered}
\operatorname{ch} L_{l k}=\operatorname{ch} V_{l k}+\sum_{j>k}\left(\operatorname{ch} V_{0 j}+\operatorname{ch} V_{1 j}\right)-\sum_{j \geqq k}\left(\operatorname{ch} V_{0 j}^{\prime}+\operatorname{ch} V_{1 j}^{\prime}\right), \quad l=0,1, \\
\operatorname{ch} L_{l k}^{\prime}=\operatorname{ch} V_{l k}^{\prime}+\sum_{j>k}\left(\operatorname{ch} V_{0 j}^{\prime}+\operatorname{ch} V_{1 j}^{\prime}-\operatorname{ch} V_{0 j}-\operatorname{ch} V_{1 j}\right), \quad l=0,1 .
\end{gathered}
$$

For $\hat{R}$ and $h=c / 16, L_{l k}, L_{l k}^{\prime}, V_{l k}, V_{l k}^{\prime} \rightarrow \tilde{L}_{l k}, \tilde{L}_{l k}^{\prime}, \widetilde{V}_{l 1}, \tilde{V}_{l k}^{\prime}$ and formulae (29) hold again.

Note that in the case $q=p+\frac{1}{v}(q=p+1$ for $\hat{W}, q=p+2$ for $\hat{S}, \hat{R})$ the modules $L_{00}=L_{10}$ [parametrized by $(m, n)$ as in Table 2] and $\tilde{L}_{00}=\tilde{L}_{10}$ for $\hat{R}$ and $(m, n)=\left(p_{12}, q_{12}\right)$ form the $c<1$ series of unitarizable HWM over $\hat{W}, \hat{S}, \hat{R}[17]$.

In the $N_{+}^{1}$ case we denote by $L_{l k}^{+}, L_{l k}^{++},(l=0,1)$, the irreducible factor-modules of $V_{l k}^{+}, V_{l k}^{\prime+}$ respectively, where $V_{l k}^{+}, V_{l k}^{\prime+}$ are given by (28) with the change $\tilde{p} \rightarrow-\tilde{p}$, $m \rightarrow-m$ (or $\tilde{q} \rightarrow-\tilde{q}, n \rightarrow-n), h \rightarrow h_{(m, n)}^{+}$given by (14) (cf. $\left.[19,21,25]\right)$. Then we have [23-25], (35):

$$
\begin{aligned}
\operatorname{ch} L_{l k}^{+}= & \operatorname{ch} V_{l k}^{+}+\sum_{j=1}^{k-1}\left(\operatorname{ch} V_{0 j}+\operatorname{ch} V_{1 j}\right)+\operatorname{ch} L_{00}^{+} \\
& -\sum_{j=0}^{k-1}\left(\operatorname{ch} V_{0 j}^{\prime}+\operatorname{ch} V_{1 j}^{\prime}\right), \quad l=0,1, k>0, \\
\operatorname{ch} L_{l k}^{++}= & \operatorname{ch} V_{l k}^{\prime+}+\sum_{j=0}^{k-1}\left(\operatorname{ch} V_{0 j}^{\prime}+\operatorname{ch} V_{1 j}^{\prime}\right)-\operatorname{ch} L_{00}^{+} \\
& -\sum_{j=1}^{k}\left(\operatorname{ch} V_{0 j}+\operatorname{ch} V_{1 j}\right), \quad l=0,1, k \geqq 0
\end{aligned}
$$

$\left(\operatorname{ch} L_{00}^{+}=\operatorname{ch} V_{00}^{+}\right.$, since $V_{00}^{+}$is irreducible). 
In the $N_{ \pm}^{2}$ cases let us denote by $L_{k}, L_{k}^{+}$the irreducible factor module of $V_{k}, V_{k}^{+}$ respectively. Then we have [23], [24], [25], (28), (29), (31):

$$
\operatorname{ch} L_{k}=\operatorname{ch} V_{k}-\operatorname{ch} V_{k+1}, \quad k \geqq 0,
$$

and [23], [24], [25], (42), (43), (44), (45):

$$
\operatorname{ch} L_{k}^{+}=\operatorname{ch} V_{k}^{+}-\operatorname{ch} V_{k-1}^{+}, \quad k>0,
$$

$\left(\operatorname{ch} L_{0}^{+}=\operatorname{ch} V_{0}^{+}\right)$.

\section{Weyl Group for the Virasoro and $N=1$ Super-Virasoro Algebras}

Consider the following infinite abelian multiplicative group $W_{a}[8]$ generated by the symbols $w(n), n \in \frac{1}{v} N$ with the properties

$$
w(n)^{2}=1, \quad w(n) w\left(n^{\prime}\right)=w\left(n^{\prime}\right) w(n) .
$$

Thus the group $W_{a}$ consists of the elements

$$
w=w\left(n_{1}\right) \ldots w\left(n_{k}\right), \quad k \in \mathbb{N}, \quad n_{i} \in \frac{1}{v} \mathbb{Z}_{+}, \quad w(0) \equiv 1 .
$$

Such a group is called a torsion group or a $p$-group [32] (here $p=2$ ). If $w=1$ or $w=w\left(n_{1}\right) \ldots w\left(n_{k}\right), n_{i} \in \frac{1}{v} \mathbb{N}, n_{i} \neq n_{j}, i \neq j$ we shall say that $w$ is given in a reduced form. Because of (33) it is clear that every element of $W_{a}$ has a reduced form.

Let $w$ be given in a reduced form, then the length of $w$, denoted $l(w)$ is defined as follows:

$$
l(w)= \begin{cases}0 & w=1 \\ k & w=w\left(n_{1}\right) \ldots w\left(n_{k}\right), n_{i} \in \frac{1}{v} \mathbb{N}, n_{i} \neq n_{j}, i \neq j .\end{cases}
$$

The length of any element $w$ is defined as the length of its reduced form. [If $l(w)=k$, then $w$ has $k$ ! reduced forms.] We need to introduce the action of $W_{a}$ on $\hat{Q}_{0}^{*}$. We set for $w$ in a reduced form and $\lambda \in \hat{Q}_{0}^{*}$ :

$$
\begin{gathered}
1 \cdot \lambda=\lambda, \\
w \cdot \lambda=\lambda+\left(n_{1}+\ldots+n_{k}\right) \lambda_{0}, \quad w=w\left(n_{1}\right) \ldots w\left(n_{k}\right), \\
n_{i} \in \frac{1}{v} \mathbb{N}, \quad n_{i} \neq n_{j},
\end{gathered}
$$

where $\lambda_{0} \in \widehat{Q}_{0}^{*}$ is defined by

$$
\lambda_{0}\left(L_{0}\right)=1, \quad \lambda_{0}(z)=0 .
$$

Setting as usual $\lambda\left(L_{0}\right)=h, \lambda(z)=c$ we shall write (36) equivalently as

$$
1 \cdot(h, c)=(h, c), \quad w \cdot(h, c)=\left(h+n_{1}+\ldots+n_{k}, c\right) \equiv(w \cdot h, c) .
$$


Note that this action is non-associative; however, this is not essential for our purposes.

Definition. Let $V=V^{h, c}$ be a (generalized) Verma module over $\hat{Q}$. We shall say that an element $w \in W_{a}$ is $V$-active if either $w=1$ or $w=w\left(n_{1}\right) \ldots w\left(n_{k}\right), n_{i} \in \frac{1}{v} \mathbb{N}, n_{i} \neq n_{j}$ and there exists a chain of invariant embeddings $V^{h, c} \rightarrow V_{1} \rightarrow \ldots \rightarrow V_{k}$ (remember that the arrows point to the embedded modules), where the submodules $V_{j}$ are isomorphic to (generalized) Verma modules over $\hat{Q}: V_{j} \cong V^{h+n_{1}+\ldots+n_{j}, c}, 1 \leqq j \leqq k$. For $h=c / 16$ and $\hat{R}$ we consider $\widetilde{V}^{c / 16, c}$ instead of $V^{c / 16, c}$.

Obviously this definition can be extended to (generalized) HWM.

It is clear that $V$ is irreducible iff only $w=1$ is $V$-active. It is also clear that if $w$ as above $V$-active and $w \neq w(n)$ there are also other $V$-active elements of $W_{a}$.

Lemma. Let $w=w\left(n_{1}\right) \ldots w\left(n_{k}\right)$ be $V$-active. Let $l \in \mathbb{N}, l<k,\left\{i_{1}, \ldots, i_{l}\right\}$ be a subset of $\left\{n_{1}, \ldots, n_{k}\right\}$, such that $i_{1}<\ldots<i_{l}$, let $m_{j}=n_{i_{j-1}+1}+\ldots+n_{i_{j}}, j=1, \ldots, l, i_{0}=0$. Then $w^{\prime} \equiv w\left(i_{1}\right) \ldots w\left(i_{l}\right)$ is also V-active.

Proof. Let $V_{j}^{\prime}=V^{h+m_{1}+\ldots+m_{j}, c}$. Note that $m_{1}+\ldots+m_{j}=n_{1}+\ldots+n_{i_{j}}$. Thus $V_{j}^{\prime}=V_{i_{j}}$. Obviously $V \rightarrow V_{i_{1}} \rightarrow \ldots \rightarrow V_{i_{1}}$.

The usefulness of this notion should become clear from the following which is the main result of the paper.

Theorem. Let $L^{h, c}$ be an irreducible GHWM over $\hat{Q}$, so that $L^{h, c} \cong V^{h, c} / I^{h, c}$, where $V \equiv V^{h, c}$ is the (generalized) Verma module of $\hat{Q}$ with the same highest weight and $I^{h, c}$ is the maximal submodule of $V^{h, c}$. Then we have for the character of $L^{h, c}$,

$$
\operatorname{ch} L^{h, c}=\sum_{\substack{w \in W_{a} \\ w-V \text {-active }}}(-1)^{l(w)} \operatorname{ch} V^{w \cdot h, c},
$$

or,

$$
\operatorname{ch} L^{h, c}(t)=\operatorname{ch} V^{h, c}(t) \sum_{\substack{w \in W_{a} \\ w-V \text {-active }}}(-1)^{l(w)} t^{w \cdot h-h} .
$$

Proof. First we note that if $V^{h, c}$ is irreducible $L^{h, c}=V^{h, c}$ and $c h L^{h, c}=\operatorname{ch} V^{h, c}$ which follows from (39) - the sum involves only $w=1,(l(1)=0)$. Further we consider the different types of embeddings according to Table 1.

Case $N^{0} . V^{h+v m n, c}$ is irreducible, while for $V=V^{h, c}$ there are only two $V$-active elements $w=1$ and $w=w(v m n)$. Thus we have from (39)

$$
\operatorname{ch} L^{h, c}=\operatorname{ch} V^{h, c}-\operatorname{ch} V^{h+v m n, c},
$$

which is the correct result according to (26).

Case $N^{2}$. We consider $V_{j}$ from Table $1, j \in \mathbb{Z}_{+}, V_{j}=V^{h_{j}, c}, c=c_{p, q}^{-}$. We shall not need the explicit values of $h_{j}$ (the reader can find them in [19] formulae (15), (17), (18) for $\hat{W},[21](13),(16),(19)$ for $\hat{S}, \hat{R},[25](27),(30),(32))$ but only the fact that $h_{j}-h_{i} \in \frac{1}{v} \mathbb{N}$ for $i<j$. We introduce the following notation

$$
w_{i, j} \equiv w\left(h_{j}-h_{i}\right), \quad i, j \in \mathbb{Z}_{+}, \quad i \leqq j, \quad(w(0) \equiv 1) .
$$


It is clear that

$$
w_{i, j} \cdot h_{l}=h_{l}+h_{j}-h_{i}, \quad w_{i, j} \cdot h_{i}=h_{j} .
$$

We fix $k \in \mathbb{Z}_{+}$and consider $V_{k}$. It is clear that $w_{k, l}, l \in \mathbb{Z}_{+}, l \geqq k$, is $V_{k}$-active. We fix $l>k$. We want to enumerate all possible $V_{k}$-active elements which act on $h_{k}$ as $w_{k, l}$, i.e. $w_{k, l} \cdot h_{k}=h_{l}$. If $l=k+1$ there are no other such elements. If $l>k+1$ besides $w_{k, l}$ they are all of the form:

$$
w_{k, k_{1}} w_{k_{1}, w_{2}} \ldots w_{k_{r} l}, \quad r=1, \ldots, l-k-1, \quad k<k_{1}<\ldots<k_{r}<l,
$$

and we agree that $w_{k, l}$ is obtained for $r=0$. For fixed $r$ there are $\left(\begin{array}{c}l-k-1 \\ r\end{array}\right)$ possible choices of $k_{1}, \ldots, k_{r}$. We note that $l\left(w_{k, k_{1}}, \ldots, w_{k_{r}, l}\right)=r+1$. Thus for the character of $L_{k}=V_{k} / I_{k}$ we have

$$
\begin{aligned}
\operatorname{ch} L_{k}(t)= & {\left[1+\sum_{l=k+1}^{\infty} \sum_{r=0}^{l-k-1} \sum_{k<k_{1}<\ldots<k_{r}<l}\right.} \\
& \left.\times(-1)^{l\left(w_{k}, k_{1} \ldots w_{k_{r} l}\right)} t^{w_{k}, k_{1} \ldots w_{k_{r} l} \cdot h-h}\right] \operatorname{ch} V_{k}(t) \\
= & {\left[1+\sum_{l=k+1}^{\infty} t^{h_{l}-h_{k}} \sum_{r=0}^{l-k-1}(-1)^{r+1}\left(\begin{array}{c}
l-k-1 \\
r
\end{array}\right)\right] \operatorname{ch} V_{k}(t) } \\
= & \left(1-t^{\left.h_{k+1}-h_{k}\right)} \operatorname{ch} V_{k}(t)=\operatorname{ch} V_{k}(t)-\operatorname{ch} V_{k+1}(t)\right.
\end{aligned}
$$

which coincides with the correct formula (31).

Case $N_{+}^{2}$. We consider $V_{j}^{+}$from Table 1, $j \in \mathbb{Z}_{+}, V_{j}=V^{h_{j}^{+}, c}, c=c_{p q}^{+}$. The explicit values of $h_{j}^{+}$can be found in [19] for $\hat{W}$, in [21] for $\widehat{S}, \hat{R}$, in [25] for $\hat{Q}$, but we shall use only the fact that $h_{j}-h_{i} \in \frac{1}{v} \mathbb{N}$ for $i>j$. Instead of (41) we introduce

$$
w_{i, j}^{+} \equiv w\left(h_{j}^{+}-h_{i}^{+}\right), \quad i, j \in \mathbb{Z}_{+}, \quad i \geqq j, \quad(w(0) \equiv 1) .
$$

We apply the same reasoning for the case $N_{-}^{2}$ and obtain for $L_{k}^{+}=V_{k}^{+} / I_{k}^{+}, k>0$,

$$
\begin{aligned}
\operatorname{ch} L_{k}^{+}(t)= & {\left[1+\sum_{l=0}^{k-1} \sum_{r=0}^{l-k-1} \sum_{k>k_{1}>\ldots>k_{r}>l}\right.} \\
& \left.\times(-1)^{l\left(w_{k}^{+}, k_{1} \ldots w_{k_{r}, l}^{+}\right)} t^{w_{k}^{+}, k_{1} \ldots w_{k_{l} l}^{+} \cdot h-h}\right] \operatorname{ch} V_{k}(t) \\
= & {\left[1+\sum_{l=0}^{k-1} t^{h_{l}^{+}-h_{k}^{+}} \sum_{r=0}^{k-l-1}(-1)^{r+1}\left(\begin{array}{c}
k-l-1 \\
r
\end{array}\right)\right] \operatorname{ch} V_{k}(t) } \\
= & {\left[1-t^{h_{k-1}^{+}-h_{k}^{+}}\right] \operatorname{ch} V_{k}(t)=\operatorname{ch} V_{k}(t)-\operatorname{ch} V_{k-1}(t), \quad k>0, }
\end{aligned}
$$

which coincides with the correct formula (32). 
Case $N^{1}$. We consider $V_{0 k}, V_{1 k}, V_{0 k}^{\prime}, V_{1 k}^{\prime}$ from Table 1 , given explicitly in (28). We set [cf. (28)]

$$
\begin{gathered}
h_{0 k}=h+v k(\tilde{p} \tilde{q} k+\tilde{q} m-\tilde{p} n), \quad h_{1 k}=h+v k(\tilde{p} \tilde{q} k-\tilde{q} m+\tilde{p} n), \\
h_{0 k}^{\prime}=h+v(\tilde{q} k+m)(\tilde{p} k+n), \quad h_{1 k}^{\prime}=h+v(\tilde{q} k+\tilde{q}-m)(\tilde{p} k+\tilde{p}-n),
\end{gathered}
$$

with $h=h_{(m, n)}$ from (13).

We start with $V_{0 k}$. Let $\mathscr{A}_{0 l}^{k}, l>k, \mathscr{A}_{1 l}^{k}, l>k, \mathscr{A}_{0 l}^{\prime k}, l \geqq k, \mathscr{A}_{1 l}^{\prime k}, l \geqq k$ denote the sets of $V_{0 k}$-active elements of $W_{a}$ such that when acting on $h_{0 k}$ we obtain $h_{0 l}, h_{1 l}, h_{0 l}^{\prime}, h_{1 l}^{\prime}$ respectively.

We claim that all elements of $\mathscr{A}_{0 l}^{k}$ are obtained as follows. To every element $\tilde{w} \in \mathscr{A}_{0, l-1}^{\prime k}$ there correspond three elements of $\mathscr{A}_{0 l}^{k}$. First $w_{1}=\tilde{w} w^{\prime}$, where $w^{\prime} \cdot h_{0, l-1}^{\prime}$ $=h_{0 l}$. Next $\tilde{w}=\tilde{w}^{\prime} w(u)$, where $\tilde{w}^{\prime} \cdot h_{0 k}=\tilde{h}\left(\right.$ for $\left.l=k+1, \tilde{w}^{\prime}=1, \tilde{h}=h_{0 k}\right), w(u) \tilde{h}=h_{0, l-1}$. To $\tilde{w}$ there correspond $w_{2}=\tilde{w}^{\prime} w\left(u^{\prime}\right)$, where $w\left(u^{\prime}\right) \cdot \tilde{h}=h_{0 l}$ and $w_{3}=\tilde{w}^{\prime} w(v) w\left(v^{\prime}\right)$, where $w(v) \cdot \tilde{h}=h_{1, l-1}^{\prime}, w\left(v^{\prime}\right) \cdot h_{1, l-1}^{\prime}=h_{0 l}$. Thus $w_{i} \cdot h_{0 k}=h_{0 l}, l\left(w_{1}\right)=l\left(w_{3}\right)=l(\tilde{w})+1, l\left(w_{2}\right)$ $=l(\tilde{w})$. Proceeding analogously we find that $\left|\mathscr{A}_{0 l}^{k}\right|=3^{2(l-k)-1},\left|\mathscr{A}_{0 l}^{\prime k}\right|=3^{2(l-k)},\left|\mathscr{A}_{1 l}^{k}\right|$ $=3^{2(l-k)-1},\left|\mathscr{A}_{1 l}^{\prime k}\right|=3^{2(l-k)}$. Further, let $\mathscr{A}_{0 l}^{k+}$ respectively $\mathscr{A}_{0 l}^{k-}$ (and analogously for the other $\mathscr{A}$ 's) be the elements of $\mathscr{A}_{0 l}^{k}$ with even, respectively odd, length. (Naturally, $\left|\mathscr{A}_{0 l}^{k+}\right|+\left|\mathscr{A}_{0 l}^{k-}\right|=\left|\mathscr{A}_{0 l}^{k}\right|$.)

We claim that

$$
\begin{aligned}
\left|\mathscr{A}_{0 l}^{k+}\right|=\left|\mathscr{A}_{0 l}^{k-}\right|+1, & \left|\mathscr{A}_{1 l}^{k+}\right|=\left|\mathscr{A}_{1 l}^{k-}\right|+1, \quad l>k, \\
\left|\mathscr{A}_{0 l}^{\prime k+}\right|=\left|\mathscr{A}_{0 l}^{\prime k-}\right|-1, \quad & \left|\mathscr{A}_{1 l}^{\prime k+}\right|=\left|\mathscr{A}_{1 l}^{\prime k-}\right|-1, \quad l \geqq k .
\end{aligned}
$$

Indeed, let $\left|\mathscr{A}_{0 l}^{k+}\right|=\left|\mathscr{A}_{0 l}^{k-}\right|+1$ for some $l$. According to our reasoning above we have

$$
\begin{aligned}
& \left|\mathscr{A}_{0 l}^{\prime k+}\right|=\left|\mathscr{A}_{0 l}^{k+}\right|+2\left|\mathscr{A}_{0 l}^{k-}\right|=3\left|\mathscr{A}_{0 l}^{k-}\right|+1, \\
& \left|\mathscr{A}_{0 l}^{\prime k-}\right|=2\left|\mathscr{A}_{0 l}^{k+}\right|+\left|\mathscr{A}_{0 l}^{k-}\right|=3\left|\mathscr{A}_{0 l}^{k-}\right|+2 .
\end{aligned}
$$

It remains to note that $\left|\mathscr{A}_{0 k+1}^{k+}\right|=2,\left|\mathscr{A}_{0 k+1}^{k-}\right|=1$.

Next we substitute (50) in (39b) and we obtain for $L_{0 k}$ (the irreducible quotient of $\left.V_{0 k}\right)$,

$$
\begin{aligned}
\operatorname{ch} L_{0 k}(t)= & {\left[1-\sum_{l \geqq k}\left(t^{h_{0 l}^{\prime}-h_{0 k}^{\prime}}+t^{h_{1 l}-h_{0 k}}\right)\right.} \\
& \left.+\sum_{l>k}\left(t^{h_{0 l}-h_{0 k}}+t^{h_{1 l}-h_{0 k}}\right)\right] \operatorname{ch} V_{0 k}(t) \\
= & \operatorname{ch} V_{0 k}(t)-\sum_{l \geqq k}\left(\operatorname{ch} V_{0 l}^{\prime}(t)+\operatorname{ch} V_{1 l}^{\prime}(t)\right) \\
& +\sum_{l>k}\left(\operatorname{ch} V_{0 l}(t)+\operatorname{ch} V_{1 l}(t)\right),
\end{aligned}
$$

which coincides with the correct result (29a).

For $c h L_{1 k}, c h L_{0 k}^{\prime}, c h L_{1 k}^{\prime}$ the argument goes analogously and the correct results in (29) are obtained as claimed from (39).

Case $N_{+}^{1}$. We consider $V_{0 k}^{+}, V_{1 k}^{+}, V_{0 k}^{+}, V_{1 k}^{\prime+}$ from Table 1 given explicitly by (28) with the changes $\tilde{p} \rightarrow-\tilde{p}, m \rightarrow-m$ [as explained before Eqs. (30)]. The proof is analogous to that of Case $N_{-}^{1}$. We start with $V_{0 k}, k>0$. Let $\tilde{\mathscr{A}}_{0 l}^{k}, l<k, \tilde{\mathscr{A}}_{1 l}, 0<l<k$, $\tilde{\mathscr{A}}_{0 l}^{\prime k}, l<k, \tilde{\mathscr{A}}_{1 l}^{\prime k}, l<k$ denote the sets of $V_{0 k}$-active elements of $W_{a}$ such that when 
acting on $h_{0 k}^{+}$we obtain $h_{0 l}^{+}, h_{1 l}^{+}, h_{0 l}^{\prime}, h_{1 l}^{\prime+}$, respectively, Analogously to the $N_{-}^{1}$ case we find that $\left|\tilde{\mathscr{A}}_{1 l}^{k}\right|=3^{2(k-l)-1},\left|\tilde{\mathscr{A}}_{0 l}^{\prime k}\right|=3^{2(k-l-1)},\left|\tilde{\mathscr{A}}_{1 l}^{k}\right|=3^{2(k-l)-1},\left|\tilde{\mathscr{A}}_{0 l}^{\prime k}\right|=3^{2(k-l-1)}$. For the elements with even (respectively odd) length $\tilde{\mathscr{A}}_{0 l}^{k+}$ (respectively $\tilde{\mathscr{A}}_{0}^{k-}$ ), we obtain the same relations as (50):

$$
\begin{gathered}
\left|\tilde{\mathscr{A}}_{0 l}^{k+}\right|=\left|\tilde{\mathscr{A}}_{0 l}^{k-}\right|+1, \quad l<k, \quad\left|\tilde{\mathscr{A}}_{1 l}^{k+}\right|=\left|\tilde{\mathscr{A}}_{1 l}^{k-}\right|+1, \quad 0<l<k, \\
\left|\tilde{\mathscr{A}}_{0 l}^{\prime k+}\right|=\left|\tilde{\mathscr{A}}_{0 l}^{\prime k-}\right|-1, \quad\left|\tilde{\mathscr{A}}_{1 l}^{\prime k+}\right|=\left|\tilde{\mathscr{A}}_{1 l}^{\prime-}\right|-1, \quad l<k .
\end{gathered}
$$

Then we substitute (52) in (39b) and obtain (30a) for $c h L_{0 k}^{+}$. Analogously one obtains the rest of (30). $\mathrm{T}$ his concludes the proof of the theorem.

\section{References}

1. Kac, V.G.: Simple irreducible graded Lie algebras of finite growth. Izv. Am. SSSR (ser. mat.), 32, 1923-1967 (1968) [English translation: Math. USSR, Izv. 2, 1271-1311 (1968)]

2. Kac, V.G.: Infinite-dimensional Lie algebras and Dedekind's $\eta$-function. Funkts. Anal. Prilozh. 8(1), 77-78 (1974) [English translation: Funkt. Anal. Appl. 8, 68-70 (1974)]

3. Kac, V.G.: Representations of classical Lie superalgebras, Lecture Notes in Mathematics, vol. 676, pp. 597-626. Berlin, Heidelberg, New York: Springer 1978

4. Kac, V.G.: Infinite-dimensional Lie algebras. An introduction. Prog. Math., vol. 44. Boston: Birkhäuser 1983

5. Kac, V.G., Peterson, D.H.: Infinite-dimensional Lie algebras, theta functions and modular forms. Adv. Math. 53, 125-264 (1984)

6. Dobrev, V.K.: Multiplet classification of the indecomposable highest weight modules over affine Lie algebras and invariant differential operators: the $A_{1}^{(1)}$ example; Talk at the Conference on Algebraic Geometry and Integrable Systems (Oberwolfach, July, 1984) and ICTP, Trieste, preprint IC/85/9 (1985)

7. Dobrev, V.K.: Multiplets of Verma modules over the osp $(2,2)^{(1)}$ super-Kac-Moody algebra. Proceedings of the Symposium on Topological and Geometrical Methods in Field Theory (Espoo, Finland, June 1986), Hietariuta, J., Westerholm, J. (eds.), pp. 93-102. Singapore: World Scientific 1986, and ICTP, Trieste, Internal Report IC/86/189 (1986)

8. Dobrev, V.K.: New Weyl groups for $A_{1}^{(1)}$ and characters of singular highest weight modules, ICTP, Trieste, preprint, IC/88/97. Commun. Math. Phys. 124, 319-336 (1989)

9. Malikov, F.G., Feigin, B.L., Fuchs, D.B.: Singular vectors in Verma modules over KacMoody algebras. Funkts. Anal. Prilozh. 20, 25-37 (1986) [English translation: Funkt. Anal. Appl. 20, 103-113 (1986)

10. Gel'fand, I.M., Fuchs, D.B.: Cohomology of the algebra of vector fields on the circle. Funkts. Anal. Prilozh. 2, 92-93 (1968)

Virasoro, M.A.: Subsidiary conditions and ghosts in dual resonance models. Phys. Rev. D 1, 2933-2936 (1970)

11. Neveu, A., Schwarz, J.H.: Factorizable dual model of pions. Nucl. Phys. B 31, 86-112 (1971)

12. Ramond, P.: Dual theory of free fermions. Phys. Rev. D3, 2415-2418 (197.1)

13. Kac, V.G.: Highest weight representations of infinite-dimensional Lie algebras. In Proceedings of ICM, Helsinki, pp. 299-304 (1978)

14. Kac, V.G.: Contravariant form for infinite-dimensional Lie algebras and superalgebras. Lect. Notes in Phys., vol. 94, pp. 441-445 (1979)

15. Feigin, B.L., Fuchs, D.B.: Invariant skew symmetric differential operators on the line and Verma modules over the Virasoro algebra. Funkts. Anal. Prilozh. 16, 47-63 (1982) [English translation: Funkt. Anal. Appl. 16, 114-126 (1982)]

16. Meurman, A., Rocha-Caridi, A.: Highest weight representations of the Neveu-Schwarz and Ramond algebras. Commun. Math. Phys. 107, 263-294 (1986)

17. Friedan, D., Qiu, Z., Shenker, S.: Superconformal invariance in two dimensions and the tricritical Ising model, Phys. Lett. 151B, 37-43 (1985) 
18. Dobrev, V.K.: Multiplet classification of the reducible elementary representations of real semisomple Lie groups: The $\mathrm{SO}_{e}(p, q)$ example. Talk at the 1st National Congress of Bulgarian Physicits, Sofia (1983), INRNE Sofia preprint (1983) and Lett. Math. Phys. 9, 205-211(1985)

19. Dobrev, V.K.: Multiplets of indecomposable highest weight modules over infinitedimensional Lie algebras: The Virasoro- $A_{1}^{(1)}$ correspondence. Proceedings of the XIII International Conference on Differential Geometric Methods in Theoretical Physics, Shumen (1984). Doebner, H.D., Palev, T.D. (eds.), pp.348-370. Singapore: World Scientific 1986, and ICTP, Trieste, preprint IC/85/13 (1985)

20. Dobrev, V.K., Petkova, V.B.: On the group-theoretic approach to extended conformal supersymmetry: classification of multiplets. Lett. Math. Phys. 9, 287-298 (1985)

21. Dobrev, V.K.: Multiplet classification of the indecomposable highest weight modules over the Neveu-Schwarz and Ramond superalgebras. Lett. Math. Phys. 11, 225-234 (1986)

22. Feigin, B.L., Fuchs, D.B.: Verma modules over the Virasoro algebra, Funkts. Anal. Prilozh. 17, 91-92 (1983)

23. Rocha-Caridi, A.: Vacuum vector representations of the Virasoro algebra. In: Vertex operators in Mathematics and Physics. Lepowsky, J., Mandelstam, S., Singen, I. (eds.), pp. 451-473. Berlin, Heidelberg, New York: Springer 1985

24. Feigin, B.L., Fuchs, D.B.: Verma modules over the Virasoro algebra. Lecture Notes in Mathematics, vol. 1060, pp. 230-245 and Moscow preprint (1984)

25. Dobrev, V.K.: Characters of the irreducible highest weight modules over the Virasoro and super-Virasoro algebras. Proceedings of the 14th Winter School on Abstract Analysis, (Srni, 1986), Suppl. Rendiconti Circolo Matematico di Palermo, Serie II, No. 14 (1987), pp. 25-42; and ICTP Trieste preprint IC/86/123 (1986)

26. Kac, V.G.: Some problems of infinite-dimensional Lie algebras and their representations. Lecture Notes in Mathematics, vol. 933, pp. 117-126. Berlin, Heidelberg, New York: Springer 1982

27. Rocha-Caridi, A., Wallach, N.R.: Characters of irreducible representations of the Lie algebra of vector fields on the circle. Invent. Math. 72, 57-75 (1983)

28. Rocha-Caridi, A., Wallach, N.R.: Characters of irreducible representations of the Virasoro algebra. Math. Z. 185, 1-21 (1984)

29. Kac, V.G., Wakimoto, M.: Unitarizable highest weight modules of the Virasoro, Neveu Schwarz and Ramond algebras. In: Lecture Notes in Physics, vol. 261, pp. 345-371. Berlin, Heidelberg, New York: Springer 1986

30. Itzykson, C., Zuber, J.B.: Two-dimensional conformal invariant theories on a torus. Nucl. Phys. B 275 [FS17], 580-616 (1986)

31. Goddard, P., Kent, A., Olive, D.: Unitary representations of the Virasoro and super-Virasoro algebras. Commun. Math. Phys. 103, 105-119 (1986)

32. Fuchs, L.: Infinite abelian groups, vols. 1, 2. New York, London: Academic Press 1970 and 1973

Kaplansky, I.: Infinite abelian groups. Ann Arbor: The University of Michigan Press 1969

Communicated by L. Alvarez-Gaumé

Received September 30, 1988 